

\title{
Search for Archaean basement in the Caledonian fold belt of North-East Greenland
}

\author{
Allen P. Nutman and Feiko Kalsbeek
}

\begin{abstract}
SHRIMP U-Pb isotope data on zircon crystals from a gneiss sample near Danmarkshavn, where the presence of Archaean rocks has earlier been documented, show that the rock has undergone a complex history of igneous and metamorphic zircon growth. At least three generations of zircon are present with ages of c. $3000 \mathrm{Ma}$, c. $2725 \mathrm{Ma}$ and $1967 \pm$ $8 \mathrm{Ma}(2 \sigma)$. Apparently the rock was formed from an Archacan protolith which underwent high grade metamorphism during the early Proterozoic. Another sample from the easternmost exposures of the Caledonian basement, collected further north, yielded only early Proterozoic zircons with an age of $1963 \pm 6 \mathrm{Ma}$. Together with a SHRIMP U-Pb zircon age of $1974 \pm 17$ Ma reported earlier, these results give evidence of a major igneous and metamorphic event in North-East Greenland about $1965 \mathrm{Ma}$ ago.
\end{abstract}

A. P. N., Research School of Earth Sciences, Australian National University, G. P. O. Box 4, Canberra A. C. T. 2601, Australia.

F. K., Geological Survey of Greenland, Øster Voldgade 10, DK-1350 Copenhagen K, Denmark.

Early Precambrian orthogneisses make up a major part of the crystalline basement of the Caledonian fold belt in North-East Greenland (Henriksen, 1991; Chadwick \& Friend, 1991; Friderichsen et al., 1991). Prior to GGU's 1988-90 mapping project in this region the only reliable age determination on these rocks gave $c .3000$ Ma for a banded gneiss collected near Danmarkshavn (zircon $\mathrm{U}-\mathrm{Pb}$ and whole-rock Rb-Sr data, Steiger et al., 1976; Fig. 1). Isotopic data (Sm-Nd and Rb-Sr model ages and SHRIMP U-Pb zircon dates) collected in connection with GGU's recent work, however, showed that most of the crystalline basement of the Caledonian fold belt in NorthEast Greenland is of early Proterozoic age. No further evidence of the presence of Archaean rocks was found, although more than 30 localities were studied (Kalsbeek et al., 1993). The present SHRIMP U-Pb zircon study had two aims: first, to seek confirmation of the Archaean age of the banded gneisses at Danmarkshavn; second, since the Danmarkshavn gneiss studied by Steiger et al. (1976) was collected in the eastern part of the exposed Caledonian basement (Fig. 1), to test whether more extensive units of Archaean gneisses existed in the easternmost parts of the investigated region. For the second purpose a sample was chosen from a site $c .110 \mathrm{~km}$ north of Danmarkshavn (Fig. 1), which possibly could belong to such an Archaean crustal block.

\section{Banded gneiss from Danmarkshavn (GGU 365358)}

This sample was taken from a locality near Danmarkshavn $\left(76^{\circ} 46.1^{\prime} \mathrm{N}, 18^{\circ} 39^{\prime} \mathrm{W}\right), 2 \mathrm{~km}$ along strike north of the site where the material studied by Steiger et al. (1976) had been collected, and in the same banded gneiss. The rock is a medium-grained tonalitic gneiss with a strong planar fabric, consisting of plagioclase, quartz, biotite ( $c$. $10 \%$ ) with some hornblende and subordinate garnet, apatite, zircon and opaque minerals. The gneisses are cut by numerous metabasic dykes at high metamorphic grade (amphibolite and granulite facies). The outcrop is characterised by alternating zones of high and low strain. Within the high-strain zones both the gneisses and the matabasic dykes are strongly deformed.

GGU sample 365358 yielded a diverse population of zircons. Grains are mostly prismatic and 200 to $300 \mu \mathrm{m}$ long. All are distinctly rounded, and some have embayed prismatic faces, indicating metamorphic corrosion rather than sedimentary abrasion. Many grains have zoned, yellow or brown, partly metamict cores and clear, structureless overgrowths. Some cores are not located in the centre of their grains. Other grains are devoid of cores and consist entirely of clear, structureless zircon.

Analysis with the ion microprobe SHRIMP 1 followed 


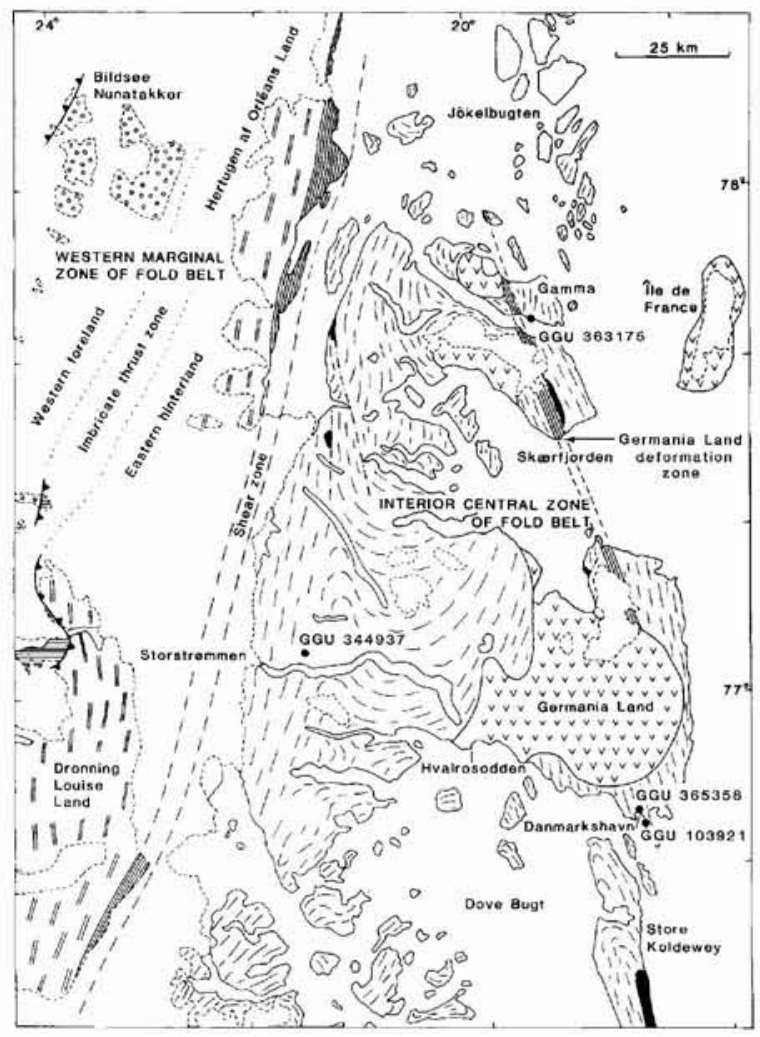

CALEDONIAN FOLO BELT



Fig. 1. Geological sketch map of the Dove Bugt - Jökelbugten region in North-East Greenland, with localities of samples mentioned in the text. GGU 103921 is the sample studied by Steiger et al. (1976).

routine analytical procedures (e.g. Compston et al., 1984; Williams \& Claesson, 1987). Thirty-six analyses were undertaken on 28 grains (Table 1A, Fig. 2A). Most of the analysed sites have moderate to high $\mathrm{U}$ content (typically $300-1000 \mathrm{ppm}$ ) but with highly variable $\mathrm{Th} / \mathrm{U}$ ratios (0.02-0.8). ${ }^{207} \mathrm{~Pb} /{ }^{206} \mathrm{~Pb}$ ages range from $c .1900$ to 3000 Ma. All analysed Archaean zircons yielded discordant ages, but some early Proterozoic zircons yielded concordant ages. The oldest zircons detected yielded ${ }^{207} \mathrm{~Pb} /{ }^{206} \mathrm{~Pb}$ ages between 2900 and $3000 \mathrm{Ma}$, and only occur as high U (900-2000 ppm) coloured, strongly zoned cores. Zircon with ${ }^{207} \mathrm{~Pb} /{ }^{206} \mathrm{~Pb}$ ages between 2600 and $2800 \mathrm{Ma}$ is structureless or weakly zoned and forms overgrowths on $>2900 \mathrm{Ma}$ zircon, whole grains and cores to Proterozoic overgrowths. The marked discordance of the Archaean zircon components (Fig. 2A) due to Proterozoic, Caledo- nian and possibly modern $\mathrm{Pb}$-loss precludes the possibility of reliable age determinations. However, the results clearly demonstrate that a $c .3000 \mathrm{Ma}$ or slightly older component is present, and that in the late Archaean either a new igneous component was added or high grade metamorphism led to zircon growth in a c. 3000 Ma rock.

Analysed Proterozoic zircon is generally clearer and less zoned than Archaean zircon. A group of analyses which consist of both whole grains and overgrowths (commonly with low $\mathrm{Th} / \mathrm{U}$ ) on Archaean zircon are close to concordant at $c .1950 \mathrm{Ma}$, or discordant with slightly younger ${ }^{207} \mathrm{~Pb} /{ }^{206} \mathrm{~Pb}$ ages and scatter along a reference chord to $380 \mathrm{Ma}$ (the U-Th- $\mathrm{Pb}$ age of titanite from this locality, Steiger et al., 1976; Fig. 2B). The most concordant sites of this group yield a weighted mean ${ }^{207} \mathrm{~Pb} /{ }^{206} \mathrm{~Pb}$ age of $1967 \pm 8 \mathrm{Ma}$ (all precisions in this paper quoted at $2 \sigma$ level), which is interpreted as the time of a high grade metamorphic event and possibly injection of new components. A few morphologically similar sites yield somewhat older ages, and indicate that a $c$. $2100 \mathrm{Ma}$ component might also be present. No Caledonian zircon overgrowths were found. However, the $\mathrm{Pb}$ loss pattern of the $1967 \pm 8$ Ma zircons is consistent with some radiogenic $\mathrm{Pb}$ loss during the Caledonian orogeny.

\section{Tonalite from Gamma Ø (GGU sample 363175)}

The sample was collected on Gamma $\emptyset\left(77^{\circ} 44.6^{\prime} \mathrm{N}\right.$, $19^{\circ} 18^{\prime} \mathrm{W}$; Fig. 1), east of the Germania Land deformation zone (Hull \& Gilotti, 1994). It represents a tonalitic gneiss with hornblende as the main mafic mineral, and is strongly deformed with a blastomylonitic texture. GGU sample 363175 yielded a uniform population of subhedral prismatic grains, typically $200-300 \mu \mathrm{m}$ long. The grains are devoid of structure or only weakly zoned, colourless or pale brown or yellow. No clear evidence of structural cores or overgrowths could be seen optically.

Thirty-six analyses were undertaken on 24 grains (Table 1B) during two analytical sessions (see below). Even with duplicate analyses on many grains no older inherited cores were detected. All analysed sites had moderate U contents (mostly $100-250 \mathrm{ppm}$ ) and moderate $\mathrm{Th} / \mathrm{U}$ ratios (mostly $0.2-0.4$ ). On a concordia plot (Fig. 2C) all analyses form a single array in which most analyses are concordant at $c$. $1950 \mathrm{Ma}$, with the remainder slightly discordant, commonly with slightly younger ${ }^{207} \mathrm{~Pb} /{ }^{206} \mathrm{~Pb}$ ages. In the first session when 22 analyses were undertaken, there was a slight problem of overcounting ${ }^{204} \mathrm{~Pb}$, giving rise to over-correction for common $\mathrm{Pb}$ and slightly too young ${ }^{207} \mathrm{~Pb} /{ }^{206} \mathrm{~Pb}$ ages. On a ${ }^{206} \mathrm{~Pb} /{ }^{208} \mathrm{~Pb}$ versus ${ }^{238} \mathrm{U}^{232} \mathrm{Th}$ plot the data fall on a $1965 \mathrm{Ma}$ reference line, indicating that use of the ${ }^{248} \mathrm{~Pb}$ correction method for 



Fig. 2A-C. Shrimp zircon U-Pb data for two gneiss samples from the Precambrian basement of the Caledonian fold in NorthEast Greenland.

common $\mathrm{Pb}$ (Compston et al., 1984) is likely to be a valid procedure. By this method the analyses that are indistinguishable from each other yielded a weighted mean ${ }^{207} \mathrm{~Pb} /{ }^{206} \mathrm{~Pb}$ age of $1962 \pm 9 \mathrm{Ma}$, agreeing well with the ${ }^{206} \mathrm{~Pb} /{ }^{238} \mathrm{U}$ age of $1963 \pm 26 \mathrm{Ma}$. For the second analytical session the ${ }^{208} \mathrm{~Pb}$ and ${ }^{204} \mathrm{~Pb}$ correction methods gave rise to weighted mean ${ }^{207} \mathrm{~Pb} /{ }^{206} \mathrm{~Pb}$ ages indistinguishable from each other (1963 \pm 9 and $1953 \pm 9 \mathrm{Ma}$, respectively), giving extra confidence to the reliability of the calculated age from the first session. When the results of both analytical sessions are pooled, data corrected for common $\mathrm{Pb}$ using the ${ }^{208} \mathrm{~Pb}$ method give rise to a weighted mean age of $1963 \pm 6 \mathrm{Ma}$, interpreted as giving the age of crystallisation of the igneous protolith of GGU 363175 .

\section{Discussion}

The results for the Danmarkshavn sample (GGU 365358) show that this rock was formed from an Archaean protolith, in agreement with the findings of Steiger et al. (1976). These authors believed that the banded gneisses were of sedimentary origin, and it must therefore be considered whether the Archaean zircons could be clastic grains in an early Proterozoic sediment. Several observations argue against this possibility: (1) Whole-rock $\mathrm{Rb}-\mathrm{Sr}$ isotope data for different layers of the banded gneiss scatter along an Archaean (c. $3000 \mathrm{Ma}$ ) isochron (Steiger et al., 1976); (2) The investigated sample is of normal tonalitic composition and is not reminiscent of a paragneiss; (3) Zircon morphology does not suggest sedimentary abrasion (see above). We therefore regard the analysed sample as an orthogneiss, which because of the presence of Archaean zircons must be of Archaean origin. Early Proterozoic zircon apparently grew during high grade metamorphism around $1965 \mathrm{Ma}$ ago. The presence of $1965 \mathrm{Ma}$ zircons with high, 'igneous', $\mathrm{Th} / \mathrm{U}$ ratios may indicate that the rock underwent incipient melting at that time. However, this is not evident from the sample, since no distinct quartzo-feldspathic sweats are present. Moreover, the rock as a whole cannot be the product of Proterozoic anatexis of an Archaean parent because is does not have the K-rich composition of an anatectic granite.

The tonalite (GGU 363175) collected further north (age $1963 \pm 6 \mathrm{Ma}$ ) was emplaced at about the time when high-grade metamorphism took place at Danmarkshavn. Another tonalitic gneiss (GGU 344937) which has been studied earlier, yielded a comparable age $(1974 \pm 17 \mathrm{Ma}$; Kalsbeek et al., 1993).

In summary, the data provide evidence of a major igneous and metamorphic event in North-East Greenland about $1965 \mathrm{Ma}$ ago. The presence of Archaean rocks at Danmarkshavn was confirmed, but this is still the only place where such rocks have been documented in NorthEast Greenland. Caledonian metamorphism, documented by mineral isotope data (Steiger $\mathrm{et} \mathrm{al}$., 1976; Brueckner \& Gilotti, 1993; Dallmeyer \& Strachan, 1994) is in this study only recorded by small degrees of $\mathrm{Pb}$ loss from parts of the analysed zircons.

Acknowledgements. The investigated samples were collected by $\mathrm{N}$. Henriksen (GGU 365358) and J. D. Friderichsen (GGU 363175). The manuscript was reviewed by J. D. Friderichsen, J. A. Gilotti, N. Henriksen and A. K. Higgins. 
Table 1. SHRImP zircon U-Pb data for two basement gneisses, North-East Greenland

\begin{tabular}{|c|c|c|c|c|c|c|c|c|c|c|}
\hline Site & $\begin{array}{c}\mathrm{U} \\
(\mathrm{ppm})\end{array}$ & $\begin{array}{c}\text { Th } \\
(\mathrm{ppm})\end{array}$ & $\mathrm{Th} / \mathrm{U}$ & $\begin{array}{l}{ }^{204} \mathrm{~Pb} \\
\left(\mathrm{pp}^{\mathrm{b}}\right)\end{array}$ & $\begin{array}{l}\text { Common } \\
{ }^{206} \mathrm{~Pb}(\%)\end{array}$ & $\frac{{ }^{206} \mathrm{~Pb}}{{ }^{238} \mathrm{U}}$ & $\frac{{ }^{207} \mathrm{~Pb}}{{ }^{235} \mathrm{U}}$ & $\frac{{ }^{207} \mathrm{~Pb}}{{ }^{206} \mathrm{~Pb}}$ & $\begin{array}{c}\text { Age } \\
(\mathrm{Ma})^{*}\end{array}$ & $\begin{array}{l}\text { Disc. } \\
(\%)\end{array}$ \\
\hline \multicolumn{11}{|c|}{ A: GGU No 365358} \\
\hline $1-1$ & 664 & 149 & 0.22 & 9 & 0.07 & $0.344 \pm 07$ & $5.64 \pm 0.13$ & $0.1190 \pm 07$ & $1942 \pm 11$ & -2 \\
\hline $1-2$ & 967 & 125 & 0.13 & 15 & 0.09 & $0.319 \pm 07$ & $5.21 \pm 0.12$ & $0.1184 \pm 05$ & $1932 \pm 08$ & -8 \\
\hline $2-10$ & 311 & 43 & 0.14 & 9 & 0.15 & $0.350 \pm 07$ & $6.33 \pm 0.15$ & $0.1313 \pm 11$ & $2115 \pm 15$ & -9 \\
\hline $3-10$ & 363 & 8 & 0.02 & 16 & 0.17 & $0.471 \pm 10$ & $12.17 \pm 0.28$ & $0.1876 \pm 10$ & $2721 \pm 09$ & -9 \\
\hline $4-1$ & 839 & 193 & 0.23 & 22 & 0.16 & $0.296 \pm 06$ & $5.20 \pm 0.12$ & $0.1276 \pm 07$ & $2066 \pm 09$ & -19 \\
\hline $5-1$ & 445 & 27 & 0.06 & 10 & 0.12 & $0.343 \pm 07$ & $5.65 \pm 0.13$ & $0.1197 \pm 09$ & $1952 \pm 13$ & -3 \\
\hline $5-2$ & 532 & 42 & 0.08 & 14 & 0.13 & $0.366 \pm 08$ & $5.95 \pm 0.14$ & $0.1180 \pm 08$ & $1926 \pm 12$ & 4 \\
\hline $7-1$ & 477 & 279 & 0.59 & 27 & 0.27 & $0.387 \pm 08$ & $9.65 \pm 0.22$ & $0.1808 \pm 10$ & $2660 \pm 09$ & -21 \\
\hline $8-1$ & 1167 & 209 & 0.18 & $<1$ & $<0.01$ & $0.315 \pm 07$ & $4.97 \pm 0.11$ & $0.1146 \pm 04$ & $1873 \pm 07$ & -6 \\
\hline $9-1$ & 602 & 129 & 0.21 & 38 & 0.40 & $0.297 \pm 06$ & $4.55 \pm 0.11$ & $0.1110 \pm 08$ & $1816 \pm 13$ & -8 \\
\hline $10-1$ & 418 & 197 & 0.47 & 21 & 0.18 & $0.504 \pm 11$ & $13.13 \pm 0.29$ & $0.1890 \pm 08$ & $2733 \pm 07$ & -4 \\
\hline $11-1$ & 164 & 127 & 0.78 & 111 & 2.72 & $0.454 \pm 10$ & $12.12 \pm 0.32$ & $0.1936 \pm 24$ & $2773 \pm 20$ & -13 \\
\hline $12-1$ & 600 & 29 & 0.05 & 29 & 0.25 & $0.359 \pm 08$ & $5.73 \pm 0.13$ & $0.1160 \pm 07$ & $1895 \pm 11$ & 4 \\
\hline $13-1 \mathrm{c}$ & 1185 & 410 & 0.35 & 7 & 0.02 & $0.507 \pm 11$ & $14.88 \pm 0.32$ & $0.2131 \pm 05$ & $2929 \pm 04$ & -10 \\
\hline $13-20$ & 757 & 64 & 0.08 & 26 & 0.19 & $0.349 \pm 07$ & $5.73 \pm 0.13$ & $0.1190 \pm 06$ & $1942 \pm 09$ & -1 \\
\hline $14-1$ & 506 & 107 & 0.21 & 18 & 0.22 & $0.293 \pm 06$ & $4.59 \pm 0.11$ & $0.1135 \pm 09$ & $1856 \pm 14$ & -11 \\
\hline $15-1$ & 867 & 186 & 0.21 & 18 & 0.10 & $0.380 \pm 08$ & $9.34 \pm 0.20$ & $0.1784 \pm 06$ & $2638 \pm 05$ & -21 \\
\hline $15-2$ & 196 & 109 & 0.56 & 8 & 0.16 & $0.452 \pm 10$ & $11.27 \pm 0.27$ & $0.1809 \pm 14$ & $2661 \pm 13$ & -10 \\
\hline $16-1$ & 214 & 74 & 0.34 & 11 & 0.24 & $0.392 \pm 08$ & $9.60 \pm 0.23$ & $0.1777 \pm 13$ & $2631 \pm 12$ & -19 \\
\hline $17-1$ & 594 & 107 & 0.18 & 10 & 0.09 & $0.352 \pm 07$ & $6.00 \pm 0.13$ & $0.1235 \pm 06$ & $2007 \pm 09$ & -3 \\
\hline $18-1$ & 580 & 40 & 0.07 & 4 & 0.04 & $0.353 \pm 09$ & $5.94 \pm 0.16$ & $0.1219 \pm 06$ & $1984 \pm 09$ & -2 \\
\hline $19-1$ & 1727 & 105 & 0.06 & 18 & 0.07 & $0.270 \pm 07$ & $4.69 \pm 0.12$ & $0.1263 \pm 04$ & $2047 \pm 06$ & -25 \\
\hline $20-1 \mathrm{c}$ & 1768 & 585 & 0.33 & 13 & 0.03 & $0.519 \pm 13$ & $15.38 \pm 0.39$ & $0.2148 \pm 04$ & $2942 \pm 03$ & -8 \\
\hline $20-20$ & 899 & 39 & 0.04 & 18 & 0.09 & $0.396 \pm 10$ & $9.81 \pm 0.25$ & $0.1797 \pm 06$ & $2650 \pm 06$ & -47 \\
\hline $21-2 c$ & 176 & 83 & 0.47 & 20 & 0.09 & $0.240 \pm 06$ & $5.93 \pm 0.18$ & $0.1790 \pm 25$ & $2643 \pm 23$ & -47 \\
\hline $21-20$ & 403 & 53 & 0.13 & 4 & 0.05 & $0.327 \pm 08$ & $5.20 \pm 0.14$ & $0.1154 \pm 08$ & $1886 \pm 12$ & -3 \\
\hline $22-1 \mathrm{c}$ & 915 & 281 & 0.31 & 78 & 0.30 & $0.525 \pm 13$ & $15.07 \pm 0.39$ & $0.2080 \pm 06$ & $2890 \pm 05$ & -6 \\
\hline $22-20$ & 373 & 65 & 0.17 & 10 & 0.13 & $0.389 \pm 10$ & $6.88 \pm 0.18$ & $0.1283 \pm 08$ & $2075 \pm 11$ & 2 \\
\hline $23-1$ & 332 & 66 & 0.20 & 11 & 0.16 & $0.373 \pm 09$ & $6.85 \pm 0.19$ & $0.1332 \pm 10$ & $2141 \pm 13$ & -5 \\
\hline $24-1$ & 1237 & 250 & 0.20 & 35 & 0.11 & $0.470 \pm 12$ & $14.49 \pm 0.37$ & $0.2235 \pm 05$ & $3006 \pm 04$ & -17 \\
\hline $25-1 c$ & 548 & 183 & 0.33 & 31 & 0.21 & $0.498 \pm 13$ & $13.75 \pm 0.36$ & $0.2003 \pm 08$ & $2828 \pm 06$ & -8 \\
\hline $25-20$ & 505 & 55 & 0.11 & 24 & 0.18 & $0.488 \pm 12$ & $12.90 \pm 0.34$ & $0.1918 \pm 08$ & $2758 \pm 07$ & -7 \\
\hline $26-1 c$ & 2310 & 412 & 0.18 & 29 & 0.10 & $0.234 \pm 06$ & $5.62 \pm 0.14$ & $0.1744 \pm 05$ & $2600 \pm 05$ & -15 \\
\hline $26-20$ & 608 & 156 & 0.26 & 30 & 0.32 & $0.281 \pm 07$ & $4.43 \pm 0.12$ & $0.1143 \pm 08$ & $1868 \pm 13$ & -15 \\
\hline $27-1$ & 373 & 121 & 0.33 & 20 & 0.24 & $0.425 \pm 11$ & $10.38 \pm 0.28$ & $0.1774 \pm 10$ & $2628 \pm 09$ & -13 \\
\hline $28-1$ & 288 & 21 & 0.07 & 21 & 0.38 & $0.364 \pm 09$ & $5.91 \pm 0.17$ & $0.1177 \pm 11$ & $1921 \pm 17$ & 4 \\
\hline \multicolumn{9}{|c|}{ B: $G G U$ No $363175^{* *}$} & \\
\hline $1-1$ & 227 & 73 & 0.32 & 17 & 0.38 & $0.359 \pm 07$ & $5.94 \pm 0.14$ & $0.1201 \pm 11$ & $1958 \pm 17$ & 1 \\
\hline $1-2$ & 208 & 61 & 0.29 & 10 & 0.25 & $0.351 \pm 06$ & $5.81 \pm 0.12$ & $0.1199 \pm 09$ & $1954 \pm 13$ & 1 \\
\hline $2-1$ & 135 & 34 & 0.25 & 11 & 0.44 & $0.337 \pm 07$ & $5.50 \pm 0.14$ & $0.1183 \pm 14$ & $1931 \pm 21$ & -3 \\
\hline $3-1$ & 224 & 69 & 0.31 & 6 & 0.15 & $0.361 \pm 07$ & $5.82 \pm 0.14$ & $0.1168 \pm 11$ & $1907 \pm 16$ & 4 \\
\hline $4-1$ & 171 & 40 & 0.24 & 11 & 0.34 & $0.363 \pm 08$ & $6.05 \pm 0.14$ & $0.1208 \pm 12$ & $1971 \pm 17$ & 1 \\
\hline 4-2 & 206 & 61 & 0.30 & 11 & 0.27 & $0.345 \pm 06$ & $5.69 \pm 0.11$ & $0.1197 \pm 09$ & $1951 \pm 13$ & -2 \\
\hline $5-1$ & 212 & 71 & 0.34 & 17 & 0.39 & $0.376 \pm 08$ & $6.22 \pm 0.15$ & $0.1202 \pm 11$ & $1959 \pm 16$ & 5 \\
\hline $5-2$ & 217 & 76 & 0.35 & 8 & 0.22 & $0.313 \pm 06$ & $4.93 \pm 0.10$ & $0.1144 \pm 09$ & $1870 \pm 15$ & -6 \\
\hline $6-1$ & 162 & 33 & 0.20 & 5 & 0.16 & $0.381 \pm 08$ & $6.37 \pm 0.15$ & $0.1211 \pm 12$ & $1972 \pm 17$ & 6 \\
\hline $6-2$ & 163 & 34 & 0.21 & 8 & 0.24 & $0.352 \pm 06$ & $5.91 \pm 0.12$ & $0.1216 \pm 08$ & $1980 \pm 12$ & -2 \\
\hline $7-1$ & 380 & 148 & 0.39 & 19 & 0.26 & $0.352 \pm 11$ & $5.90 \pm 0.19$ & $0.1215 \pm 10$ & $1979 \pm 14$ & -2 \\
\hline $8-1$ & 164 & 36 & 0.22 & 26 & 0.91 & $0.324 \pm 10$ & $5.37 \pm 0.18$ & $0.1203 \pm 15$ & $1960 \pm 22$ & -8 \\
\hline $8-2$ & 269 & 77 & 0.29 & 11 & 0.22 & $0.333 \pm 06$ & $5.40 \pm 0.10$ & $0.1178 \pm 08$ & $1924 \pm 12$ & -4 \\
\hline $9-1$ & 222 & 52 & 0.23 & 25 & 0.59 & $0.354 \pm 11$ & $5.94 \pm 0.20$ & $0.1217 \pm 12$ & $1982 \pm 17$ & -1 \\
\hline $9-2$ & 173 & 58 & 0.33 & 14 & 0.44 & $0.324 \pm 06$ & $5.26 \pm 0.11$ & $0.1178 \pm 10$ & $1923 \pm 16$ & -6 \\
\hline $10-1$ & 136 & 29 & 0.21 & 24 & 0.96 & $0.344 \pm 11$ & $5.63 \pm 0.20$ & $0.1189 \pm 15$ & $1939 \pm 23$ & -2 \\
\hline
\end{tabular}


Table 1 continued

\begin{tabular}{|c|c|c|c|c|c|c|c|c|c|c|}
\hline Site & $\begin{array}{c}U \\
\text { (ppm) }\end{array}$ & $\begin{array}{c}\mathrm{Th} \\
(\mathrm{ppm})\end{array}$ & $\mathrm{Th} / \mathrm{U}$ & $\begin{array}{l}{ }^{204} \mathrm{~Pb} \\
\left(\mathrm{pp}^{\mathrm{b}}\right)\end{array}$ & $\begin{array}{l}\text { Common } \\
{ }^{206} \mathrm{~Pb}(\%)\end{array}$ & $\frac{{ }^{206} \mathrm{~Pb}}{{ }^{238} \mathrm{U}}$ & $\frac{{ }^{207} \mathrm{~Pb}}{{ }^{235} \mathrm{U}}$ & $\frac{{ }^{207} \mathrm{~Pb}}{{ }^{206} \mathrm{~Pb}}$ & $\begin{array}{c}\text { Age } \\
(\mathrm{Ma})^{*}\end{array}$ & $\begin{array}{c}\text { Disc. } \\
(\%)\end{array}$ \\
\hline $11-1$ & 260 & 53 & 0.21 & 25 & 0.50 & $0.355 \pm 11$ & $6.04 \pm 0.20$ & $0.1234 \pm 11$ & $2006 \pm 15$ & -2 \\
\hline $11-2$ & 212 & 45 & 0.21 & 8 & 0.19 & $0.347 \pm 06$ & $5.84 \pm 0.11$ & $0.1221 \pm 08$ & $1988 \pm 11$ & -3 \\
\hline $12-1$ & 217 & 62 & 0.28 & 6 & 0.17 & $0.318 \pm 10$ & $5.37 \pm 0.18$ & $0.1226 \pm 12$ & $1995 \pm 18$ & -11 \\
\hline $12-2$ & 223 & 72 & 0.32 & 16 & 0.36 & $0.350 \pm 06$ & $5.78 \pm 0.11$ & $0.1199 \pm 08$ & $1954 \pm 11$ & -1 \\
\hline $13-1$ & 185 & 39 & 0.21 & 15 & 0.50 & $0.298 \pm 09$ & $4.88 \pm 0.16$ & $0.1189 \pm 13$ & $1940 \pm 20$ & -13 \\
\hline $13-2$ & 317 & 84 & 0.27 & 18 & 0.28 & $0.345 \pm 06$ & $5.64 \pm 0.11$ & $0.1184 \pm 07$ & $1932 \pm 11$ & -1 \\
\hline $14-1$ & 196 & 39 & 0.20 & 26 & 0.73 & $0.334 \pm 10$ & $5.50 \pm 0.18$ & $0.1195 \pm 12$ & $1949 \pm 18$ & -5 \\
\hline $14-2$ & 144 & 42 & 0.29 & 13 & 0.50 & $0.313 \pm 06$ & $5.10 \pm 0.11$ & $0.1181 \pm 11$ & $1928 \pm 17$ & -9 \\
\hline $15-1$ & 255 & 97 & 0.38 & 18 & 0.38 & $0.344 \pm 10$ & $5.78 \pm 0.19$ & $0.1218 \pm 12$ & $1983 \pm 17$ & -4 \\
\hline $16-1$ & 159 & 42 & 0.27 & 14 & 0.46 & $0.359 \pm 11$ & $5.93 \pm 0.20$ & $0.1199 \pm 13$ & $1954 \pm 20$ & 1 \\
\hline $16-2$ & 164 & 53 & 0.32 & 12 & 0.39 & $0.347 \pm 06$ & $5.78 \pm 0.12$ & $0.1208 \pm 09$ & $1968 \pm 13$ & -2 \\
\hline $17-1$ & 111 & 23 & 0.21 & 17 & 0.90 & $0.325 \pm 10$ & $5.36 \pm 0.19$ & $0.1195 \pm 15$ & $1948 \pm 23$ & -7 \\
\hline $18-1$ & 100 & 19 & 0.19 & 15 & 0.88 & $0.318 \pm 10$ & $5.09 \pm 0.18$ & $0.1161 \pm 17$ & $1898 \pm 26$ & -6 \\
\hline 19-1 & 152 & 38 & 0.25 & 34 & 1.23 & $0.331 \pm 10$ & $5.40 \pm 0.18$ & $0.1183 \pm 14$ & $1930 \pm 21$ & -5 \\
\hline $19-2$ & 212 & 63 & 0.30 & 15 & 0.37 & $0.330 \pm 06$ & $5.41 \pm 0.11$ & $0.1188 \pm 09$ & $1938 \pm 13$ & -5 \\
\hline $20-1$ & 247 & 52 & 0.21 & 23 & 0.49 & $0.345 \pm 10$ & $5.71 \pm 0.19$ & $0.1200 \pm 10$ & $1956 \pm 15$ & -2 \\
\hline $21-1$ & 226 & 62 & 0.28 & 26 & 0.59 & $0.360 \pm 11$ & $5.75 \pm 0.19$ & $0.1157 \pm 11$ & $1890 \pm 16$ & 5 \\
\hline $22-1$ & 488 & 125 & 0.26 & 23 & 0.24 & $0.359 \pm 11$ & $6.11 \pm 0.19$ & $0.1234 \pm 07$ & $2006 \pm 10$ & -1 \\
\hline $23-1$ & 233 & 64 & 0.27 & 10 & 0.23 & $0.341 \pm 06$ & $5.66 \pm 0.11$ & $0.1206 \pm 07$ & $1965 \pm 11$ & -4 \\
\hline $24-1$ & 149 & 49 & 0.33 & 35 & 1.41 & $0.290 \pm 05$ & $4.47 \pm 0.10$ & $0.1118 \pm 12$ & $1828 \pm 19$ & -10 \\
\hline
\end{tabular}

Notes: ${ }^{* 207} \mathrm{~Pb} /{ }^{206} \mathrm{~Pb}$ ages corrected for very small amounts of common lead. Uncertainties are given at the $1 \sigma$ level. ${ }^{* * 20 \%} \mathrm{~Pb}$ correction method employed instead of ${ }^{204} \mathrm{~Pb}$ method, see text for explanation. o and $\mathrm{c}$, visible structural overgrowths and cores, respectively. Disc. (\%): discordance calculated as $100 \times\left[\left(\left(^{206} \mathrm{~Pb} /{ }^{238} \mathrm{U}\right.\right.\right.$ age $) /\left({ }^{207} \mathrm{~Pb} /{ }^{206} \mathrm{~Pb}\right.$ age $\left.)-1\right]$.

\section{References}

Brueckner, H. K. \& Gilotti, J. A. 1993: Preliminary age constraints on the timing of eclogite facies metamorphism, North-East Greenland Caledonides. Geol. Soc. Am. Abstr. with Programs 25, A340 only.

Chadwick, B. \& Friend, C. R. L. 1991: The high-grade gneisses in the south-west of Dove Bugt: an old gneiss complex in a deep part of the Caledonides of North-East Greenland. Rapp. Gronlands geol. Unders. 152, 103-111.

Compston, W. Williams, I. S. \& Meyer, C. 1984: U-Pb geochronology of zircons from Lunar Breccia 73217 using a sensitive high mass-resolution ion microprobe. Proc. 14th Lunar Planet. Sci. Conf., J. geophys. Res. Suppl. 89, B525-534.

Dallmeyer, R. D. \& Strachan, R. A. $1994:{ }^{40} \mathrm{Ar} /{ }^{39} \mathrm{Ar}$ mineral age constraints on the timing of deformation and metamorphism, North-East Greenland Caledonides. Rapp. Gronlands geol. Unders. 162, (this volume).

Friderichsen, J. D., Gilotti, J. A., Henriksen, N., Higgins, A. K., Hull, J. M., Jepsen, H. F. \& Kalsbeek, F. 1991: The crystalline rocks of Germania Land, Nordmarken and adjacent areas, North-East Greenland. Rapp. Gronlands geol. Unders. 152, 85-94.

Henriksen, N. 1991: The North-East Greenland project 19881990. Rapp. Grønlands geol. Unders. 152, 24-29.

Hull, J. M. \& Gilotti, J. A. 1994: The Germania Land deformation zone and related structures, North-East Greenland. Rapp. Gronlands geol. Unders. 162 (this volume).

Kalsbeek, F., Nutman, A. P. \& Taylor, P. N. 1993: Palaeoproterozoic basement province in the Caledonian fold belt of North-East Greenland. Precambrian Res. 63, 163-178.

Steiger, R. H., Harnik-Soptrajanova, G., Zimmerman, E. \& Henriksen, N. 1976: Isotopic age and metamorphic history of the banded gneiss at Danmarkshavn, East Greenland. Contr. Mineral. Petrol. 57, 1-24.

Williams, I. S. \& Claesson, S. 1987: Isotopic evidence for the Precambrian provenance and Caledonian metamorphism of the high-grade paragneisses from the Seve Nappes, Scandinavian Caledonides, II. Ion microprobe zircon U-Th- $\mathrm{Pb}$. Contr. Mineral. Petrol. 97, 205-217. 